\title{
Congenital Midgut Malrotation Presenting as Acute Duodenal Obstruction in an Adult_-Laparoscopic Management
}

\author{
Rafique U Harvitkar ${ }^{1}$, Abhijit Joshi
}

\begin{abstract}
Background: Intestinal malrotation is a congenital anomaly, wherein the midgut fails to rotate completely or partially during the early embryological developmental phase. The rotation is usually counterclockwise at $270^{\circ}$ around the axis of the superior mesenteric artery (SMA). Malrotation is most commonly seen in pediatric population with the majority of patients presenting in their early childhood before the first year of life. Acute duodenal obstruction due to midgut malrotation in an adult is a rare manifestation. Therefore, midgut malrotation should be the differential diagnosis in an adult with bowel obstruction.

Case description: We present a case of a 26-year-old male who presented with acute-onset abdominal pain with multiple episodes of bilious vomiting. Contrast-enhanced computed tomography of the abdomen (CECT) revealed intestinal malrotation with all parts of the duodenum (D1-D4) toward the right of the midline. The patient underwent an emergency laparoscopic Ladd's procedure. Postoperative recovery was uneventful.

Conclusion: Adult patients with vague abdominal symptoms should raise a high index of suspicion for malrotation. An early and prompt diagnosis will prevent fatal complications associated with this disease and can be managed laparoscopically by Ladd's procedure.

Keywords: D1 to D4 of the duodenum, Embryologic development, Intestinal malrotation, Ladd's band, Ladd's procedure.

World Journal of Laparoscopic Surgery (2021): 10.5005/jp-journals-10033-1439
\end{abstract}

\section{INTRODUCTION}

Intestinal malrotation is a congenital anomaly, wherein the midgut fails to rotate completely or incompletely during the early embryological developmental phase. Developmental failure of the ligament of Treitz leads to the presence of duodenojejunal flexure (DJ) along with the small bowel toward the right side of the vertebrae. Intestinal malrotation affects 1 in every 500 neonates, and $65-85 \%$ of malrotation cases present within 4 weeks of life. ${ }^{1,2}$ However, $90 \%$ of cases present within the first year of life. ${ }^{3}$

Intestinal malrotation presenting in an adult is a very rare entity. It accounts for $0.0002 \%$ to $0.02 \%$ of all cases of intestinal malrotations. ${ }^{3}$ Most of these cases are asymptomatic and diagnosed incidentally on radiological imaging or unrelated surgery. Hence, it is difficult to determine the exact counts of patients with malrotation.

However, a few of these patients may present with acute or chronic symptoms. Acute symptoms include nausea, vomiting, abdominal pain, abdominal distension, constipation, or obstipation. Whereas, chronic symptoms include intermittent dull aching or crampy abdominal pain and altered bowel habits (diarrhea/ constipation) with general weakness. The surgeon should be vigilant while evaluating an adult patient with acute or chronic symptoms of intestinal obstruction. Undiagnosed cases can lead to fatal complications, such as bowel ischemia or necrosis.

In 1932, Ladd was the first to discover the peritoneal bands responsible for midgut malrotation, hence named Ladd's bands. ${ }^{4}$ We herein report a case of acute intestinal (duodenal) obstruction caused by midgut malrotation.

\section{Case Description}

A 26-year-old male with no comorbidities was admitted to the emergency ward with acute-onset abdominal pain and multiple

\footnotetext{
1,2 Department of General and Laparoscopic Surgery, Dr LH Hiranandani Hospital, Mumbai, Maharashtra, India

Corresponding Author: Rafique U Harvitkar, Department of General and Laparoscopic Surgery, Dr LH Hiranandani Hospital, Mumbai, Maharashtra, India, Phone: +91 8879525195, e-mail: dr_rafique639@ yahoo.com

How to cite this article: Harvitkar RU, Joshi A. Congenital Midgut Malrotation Presenting as Acute Duodenal Obstruction in an AdultLaparoscopic Management. World J Lap Surg 2021; 14(1):61-64.

Source of support: Nil

Conflict of interest: None
}

episodes of bilious vomiting for 2 days. For 2 years, he had intermittent dull aching epigastric pain, diagnosed as "chronic gastritis."

The patient did not experience fever, altered bowel habits, or melena. He did not give any history of previous surgeries. He was hemodynamically stable.

Physical examination revealed abdominal distension, epigastric tenderness, and an empty rectum. The patient was admitted for further evaluation with a provisional diagnosis of acute exacerbation of chronic gastritis. He was kept nil by mouth and started on intravenous fluids, proton pump inhibitors, antiemetics, and continuous nasogastric tube aspiration. His symptoms started progressively worsening despite 2 days of conservative medical management. Hence, he was investigated further. Routine blood investigations were unremarkable.

\section{Radiological imaging:}

- The erect abdominal X-ray revealed a "double-bubble sign." 

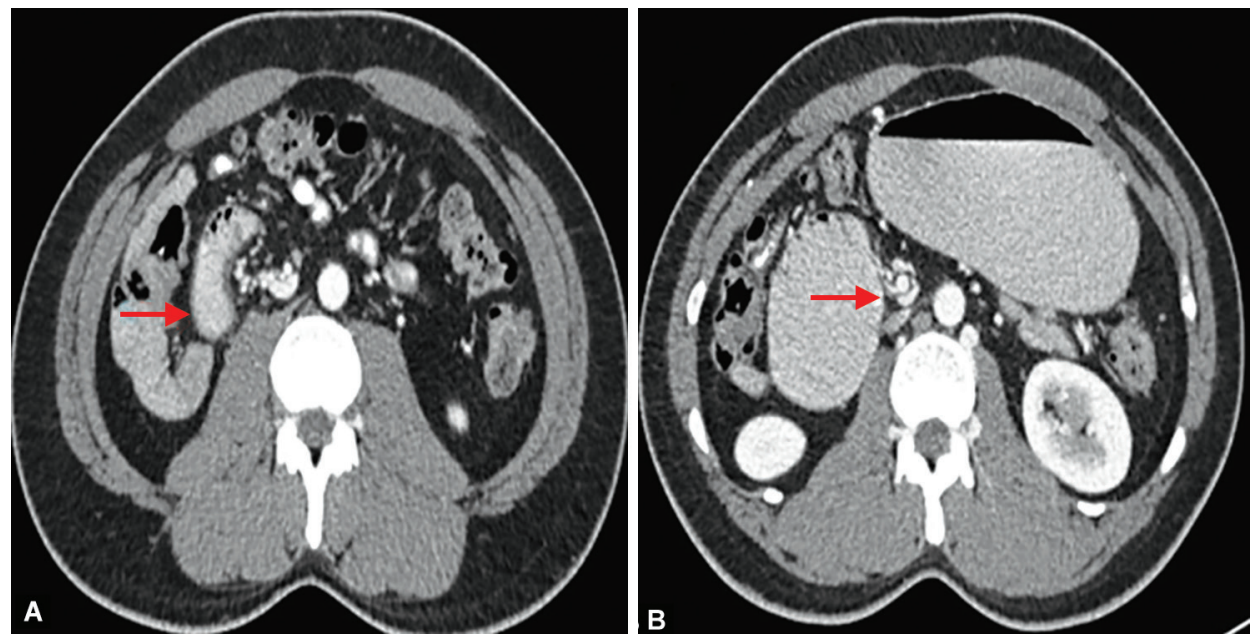

Figs. $1 \mathrm{~A}$ and B: CT scan of the abdomen: (A) axial view showing the duodenum toward right of the vertebral column (B) "whirlpool sign" of mesenteric vessels
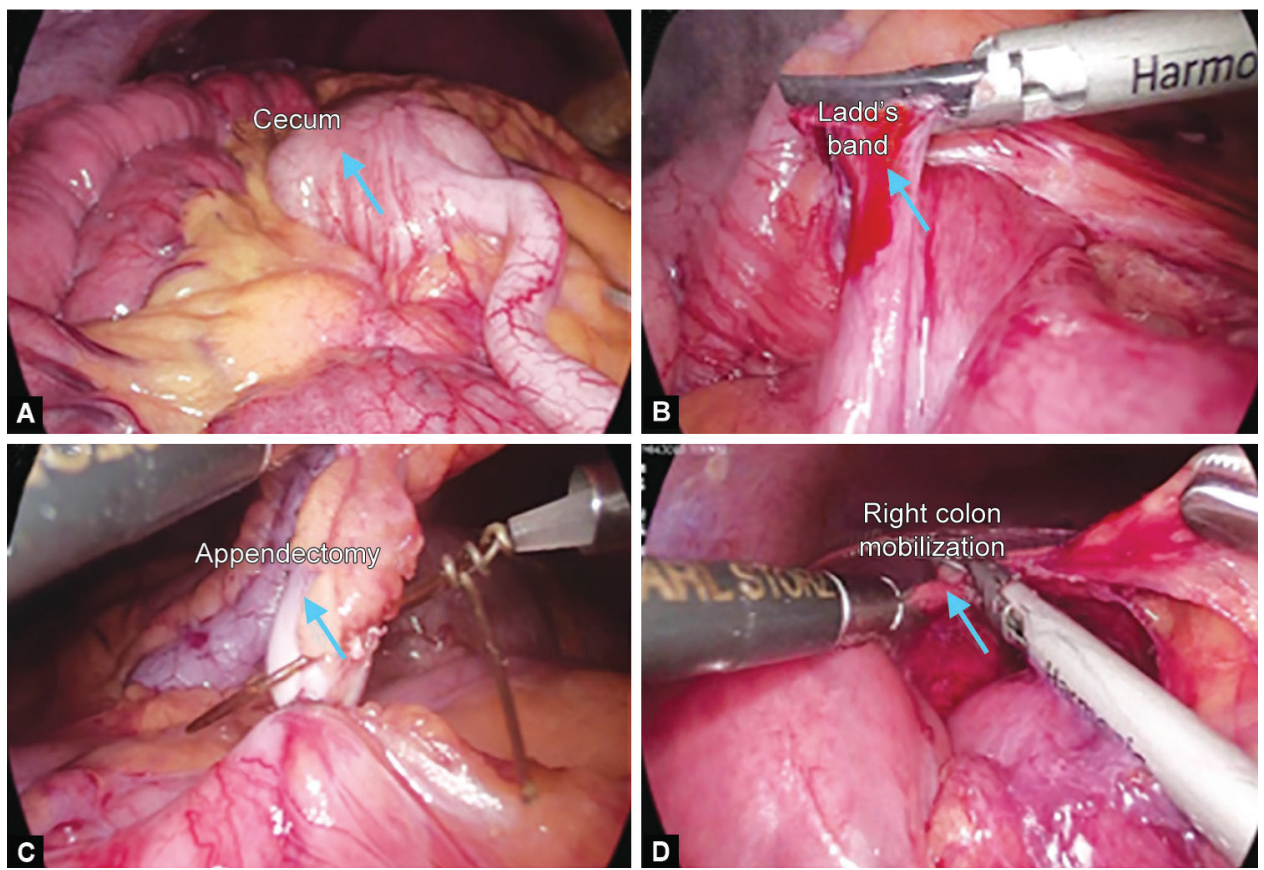

Figs. 2A to D: Laparoscopic view: (A) abnormally located cecum with the appendix toward the left upper abdomen with the small bowel toward the right side, (B) Ladd's band being divided, (C) Appendectomy, (D) Right colon mobilization medially along the hepatic flexure

- Contrast-enhanced computed tomography (CECT) of the abdomen revealed:

o Gross distension of the stomach and duodenum.

o The third and fourth parts of the duodenum were not seen, traversing toward the left side of the spine with the DJ seen orienting to the right of the midline (Fig. 1A). The caliber of the small bowel immediately distal to the distended duodenum appears normal.

o Swirling of the root of the mesentery and superior mesenteric vein (SMV) seen around the super mesenteric artery (SMA) in a counterclockwise fashion, suggestive of "whirlpool sign" (Fig. 1B). Inverse relation of SMA and SMV was suggestive of intestinal malrotation, resulting in midgut volvulus.

Given the above radiological findings, the patient was posted for surgery. At laparoscopy, the small bowel was seen on the right side of the abdomen, whereas the appendix and cecum were seen in the left hypochondriac region. There were multiple bands seen traversing from the cecum laterally, extrinsically compressing the distal duodenum. The patient underwent laparoscopic Ladd's procedure with the following steps (Figs 2A to 2D):

- Division of Ladd's bands (Fig. 2B)

- Broadening of the small intestinal mesentery

- Appendectomy (Fig. 2C)

- Placement of the small bowel along the right gutter and colon along the left gutter

The patient tolerated the procedure well. Postoperative recovery was uneventful. He was discharged on day 3 of the surgery on a full diet.

\section{Discussion}

Intestinal malrotation is a congenital anomaly that results from incomplete or abnormal rotation of the bowel during 

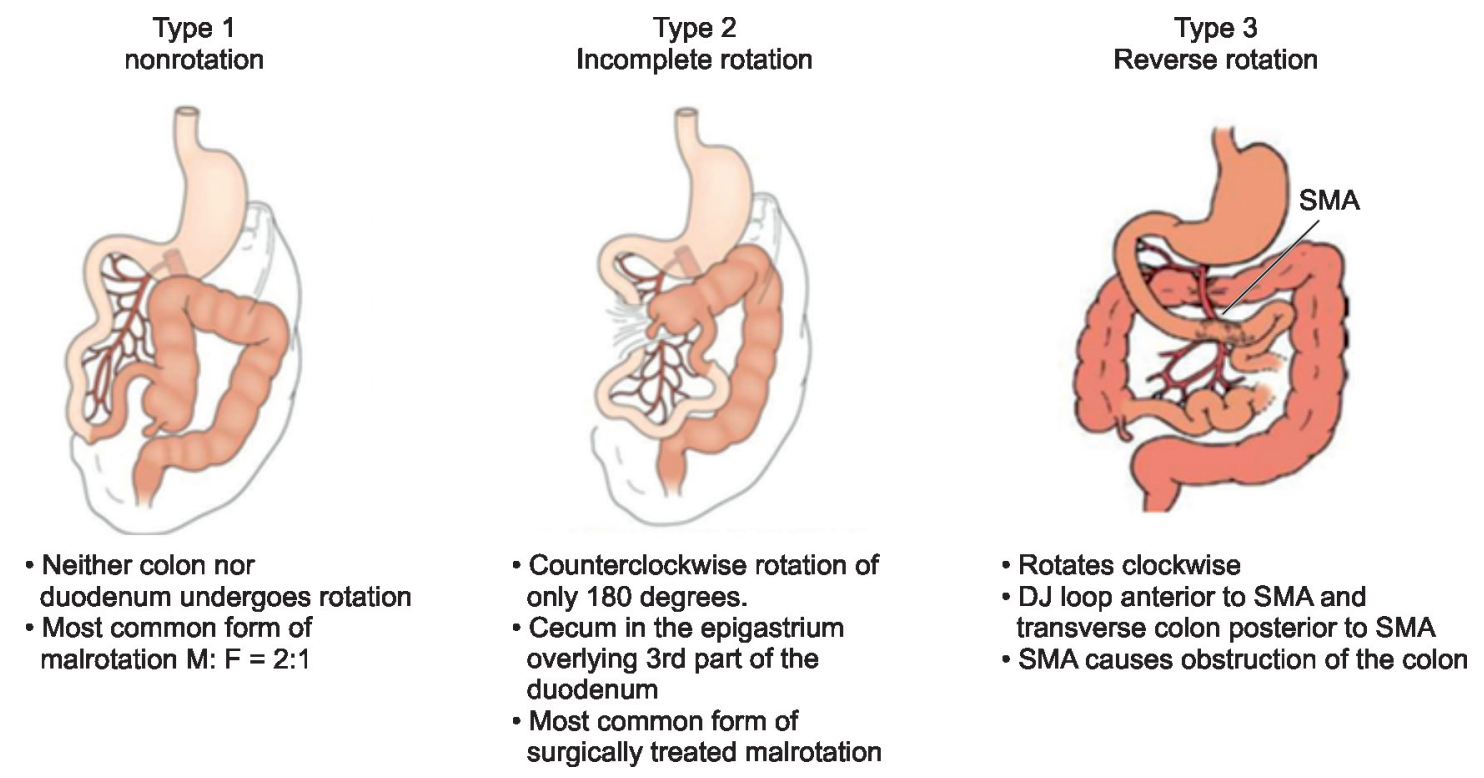

Fig. 3: Stringer classification of malrotation: type 1: nonrotation, type 2: incomplete rotation, and type 3: reverse rotation

embryogenesis. Franklin Mall was the first to describe the development and position of the human intestine in $1897 .{ }^{5}$ Stages of normal rotation include herniation, rotation, retraction, and fixation.

During normal embryonic development, the bowel protrudes into the base of the umbilical cord and promptly elongates. As it returns to the abdominal cavity, it undergoes $270^{\circ}$ counterclockwise rotation around the axis of the SMA, and as a result, the DJ is commonly located to the left of the first lumbar vertebra (L1), and the terminal ileum in the right iliac fossa. This results in a broad mesentery, running obliquely down from the DJ flexure to the cecum, avoiding rotation around SMA. ${ }^{6}$ Any deviation from normal rotation results in malrotation with the shorter root of the mesentery, making it more vulnerable to volvulus.

Stringer classified malrotation based on the embryological state of development into three main types as (Fig. 3): type I (nonrotation) here $\mathrm{DJ}$ lies on the right and the colon on the left, type II (duodenal malrotation) with the cecum in the epigastric region overlying the third part of the duodenum, and type III (combined duodenal and cecal malrotation) here DJ loop anterior to SMA and transverse colon posterior to SMA. ${ }^{7}$ Our patient had type II malrotation.

Patients with malrotation can present with acute or chronic symptoms or incidental findings on imaging/surgery. Acute manifestations (sudden-onset abdominal pain, bilious vomiting, or obstipation) may suggest midgut volvulus. However, dull aching/ crampy abdominal pain, altered bowel habits, and malabsorption are vague chronic symptoms. ${ }^{8}$

CECT scan of the abdomen is the gold standard imaging modality for adult malrotation; however, an upper gastrointestinal contrast study is reserved for the pediatric population. Computed tomography (CT) can demonstrate inversion of SMA and SMV, bowel position and viability, and volvulus (whirlpool sign) (Fig. 1). Other less common modalities include ultrasonography and magnetic resonance imaging of the abdomen. ${ }^{9}$

Patients with malrotation can be treated by Ladd's procedure. The basic principle of the surgery remains the same, irrespective of the technique (open/laparoscopy).

There are six key elements in the operative correction of malrotation via Ladd's procedure (Fig. 2). ${ }^{10}$
1. Entry into the abdominal cavity and evisceration

2. Counterclockwise detorsion of the bowel (acute cases)

3. Division of Ladd's bands

4. Broadening of the small intestine mesentery

5. Incidental appendectomy

6. Placement of the small intestine along the right flank and colon along the left flank of the abdomen

There are controversies regarding the management of incidental intestinal malrotation. The following can be considered a relative contraindication for performing the Ladd's procedure:

- Patient with asymptomatic or incidentally found rotational anomaly

- Complex cardiac disease (i.e., heterotaxy) with asymptomatic malrotation

- Older patients with chronic symptoms without volvulus

However, each of these may still warrant elective Ladd's procedure, with the risk of future volvulus as high as $20 \%$. Magnified vision, faster recovery, less hospital stay, and early mobilization are the main advantages of laparoscopic surgery over open technique. Hence, it was preferred in our case. The patient was mobilized on the same evening and discharged on day three.

\section{Conclusion}

An important feature noted in our case is our patient's previous history of dull aching, vague abdominal pain which was misdiagnosed as "chronic gastritis." This suggests that intestinal malrotation and volvulus may be worth considering, with a high index of suspicion in an adult patient presenting with chronic vague symptoms of the abdomen. The early and prompt diagnosis will prevent fatal complications associated with this disease. Malrotation can be managed laparoscopically by Ladd's procedure.

\section{Patient Consent}

Written informed consent was obtained from the patient for publication of this case and any accompanying images. 


\section{References}

1. Sahu SK, Raghuvanshi S, Sinha A, et al. Adult intestinal malrotation presenting as midgut volvulus; case report. J Surg Arts 2012;5(1): 18-21. ISSN: 1308-0709.

2. Matzke GM, Moir CR, Dozois EJ. Laparoscopic Ladd procedure for adult malrotation of the midgut with cocoon deformity: report of a case. J Laparoendosc Adv Surg Tech A 2003;13:327-329. DOI: 10.1089/109264203769681736.

3. Wang C. Welch Anomalies of intestinal rotation in adolescents and adults. Surgery 1963;54:839-855. PMID: 14087118.

4. Von Flüe M, Herzog U, Ackermann C, et al. Acute and chronic presentation of intestinal non rotation in adults. Dis Colon Rectum 1994;37(2):192-198. DOI: 10.1007/BF02047549.

5. Mall FP. Development of the human intestine and its position in the adult. Bull Johns Hopkins Hosp 1898;9:197-208. Available from: https://embryology.med.unsw.edu.au/embryology/index. php/Paper_-_Development_of_the_human_intestine_and_its_ position_in_the_adult_(1898).
6. Pickhardt PJ, Bhalla S. Intestinal malrotation in adolescents and adults: spectrum of clinical and imaging features. AJR Am J Roentgenol 2002;179(6):1429-1435. DOI: 10.2214/ajr.179.6.1791429.

7. Ben Ely A, Gorelik N, Cohen-Sivan Y, et al. Appendicitis in adults with incidental midgut malrotation: CT findings. Clin Radiol 2013;68(12):1212-1219. DOI: 10.1016/j.crad.2013.07.001.

8. Emanuwa OF, Ayantunde AA, Davies TW. Midgut malrotation first presenting as acute bowel obstruction in adulthood: a case report and literature review. World J Emerg Surg 2011;6(1):22. DOI: 10.1186/1749-7922-6-22.

9. Dietz DW, Walsh RM, Grundfest-Broniatowski S, et al. Intestinal malrotation: a rare but important cause of bowel obstruction in adults. Dis Colon Rectum 2002;45(10):1381-1386. DOI:10.1007/s10350004-6429-0.

10. Moldrem AW. Late presentation of intestinal malrotation: an argument for elective repair. World J Surg 2008;32(7):1426-1431. DOI: 10.1007/s00268-008-9490-3. 